\title{
Accelerator Power Concepts Using Isolated Transmission Lines
}

․․․ 


\title{
ACCFLERATOR POWER CONCEPTS
}

\section{USING ISOLATED TRANSMISSION LINES}

by

W. C. Nunnaliy

\section{ABSTRACT}

\begin{abstract}
This report outlines the rationale and the advantages of using multiple transmission-line sections isolated by transit time and inductance in accelerating high-current $(\simeq 10-k A)$, short-pulse ( $\leqslant 100-n s)$ particle beams to the mult imegavolt level. The main advantages of this system include reducing the number of output switches required per output pulse by nearly an order of magnitude over conventional systems and increasing the system capability for repetition-rate operation. The isolated transmission-line concept is developed, and possible modes of operation are outiined. In addition, a point design of a 10-kA short-pulse accelerator is presented.
\end{abstract}

\section{INTRODUCTION}

The accelerator power schemes currently used in directed energy systems such as the experimental test accelerator (ETA) ${ }^{1}$ and the advanced test accelerator (ATA) ${ }^{1}$ require many high-quality (highvoltage, low-inductance, loi-jitter, and highrepetition rate) switches to accelerate single pulses in sequence. These switches must be high quality because they play a primary role in the acceleration scheme.

The isolated transmission-lire accelerator (ITLA) power scheme presented here uses high-power switches in a secondary role that requires lower quality units. ETA/ATA-type acceleration power schemes use the switching action of one switch only one time, whereas the ITLA scheme uses one switch many times through transit-time isolation. The ITLA power scheme can reduce by 10 times the number of switches required by an ETA/ATA system or can provide acceleration power for up to 10 simultaneous ETA/ATA-type pulses with the same number of switches. Another advantage of the ITLA power scheme is that because the system is not limited by ferrite size as is the ETA/ATA system, it can use the higher saturation flux density and slower frequency response of tape-wound iron cores.

\section{ETA/ATA ACCELERATOR POWER SYSTEM}

The ETAIATA accelerator power system uses many pulse generator units that are transformer-coupled sequentially to the pulse being accelerated. A simplified schematic of a one-pulse generator system, which consists of a biumlein line, a large ferrite torc $d$, and a high-quality switch, is shown in Fig. 1. The Blumlein line initially is charged to voltage $v_{0}$. The main switch is closed at time $t=0$ to start the propagation of a shorting wave toward the load from $A$ to $B$. One-line transit time $T_{L}$ later, the wave appears at $B$. If the beam pulse leading edge is at $C$, the beam pulse duration is $T_{p}=$ $2 T_{L}$, and the beam puise amplitude is $I_{p}=V_{0} / 2 Z_{L}$. the Blumlein line wave experiences a matched load because of the transformer-coup led beam current. The beam pulse is accelerated through a potential of $V_{0}$ for a time equal to $2 T_{L}=T_{p}$. This method of system operation places severe requirements on the operation of the main switch. 


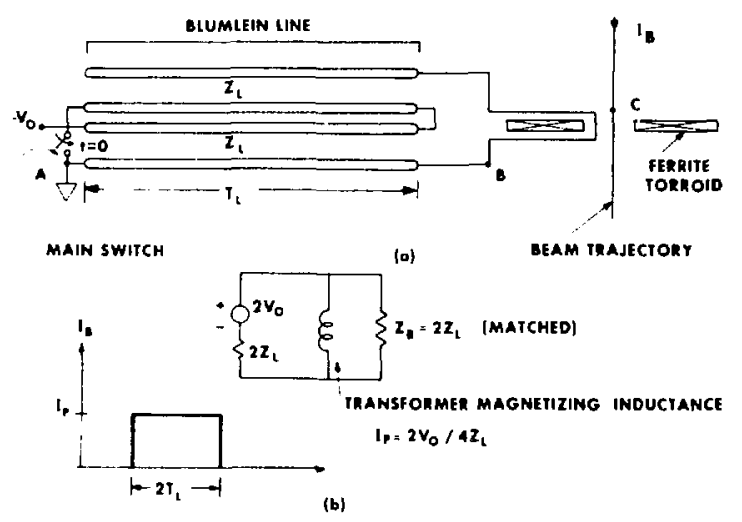

Fig. 1. ETA/ATA-type induction accelerator module.

First, the switch must be triggered with low jitter so the Blumlein line wave arrival at $B$ is synchronized with the beam pulse arrival. Second, the switch must close in a time $T_{c}$, much less than the one-way line transit time $T_{L}$. The switch closure time $T_{c}$ is determined by the switch inductance $L_{S}$ and the line impedance $Z_{L}$, or $T_{C} \simeq 3 L_{S} Z_{L} \ll T_{L} /$ 10. Thus, the switch inductance must be low to permit fast-current risetimes in low-impedance circuits. In addition, the switch also must have a high repetition-rate capability because the switch must be triggered one time for each pulse to be accelerated.

\section{ITLA POWER SYSTEM}

The ITLA power concept will be developed with a progression of circuits and applications. A basic charged transmission-line accelerator is shown in Fig. 2. This transmission line with impedance $L_{L}$ is charged to voitage $V_{0}$ and will accelerate a matched beam current pulse of $I_{B}=V_{0} / 22$ and pulse length of $T_{p}=2 T_{L}$ to $\mathrm{on}$ additional potential of $v_{0} / 2$ (assuming the beam initially is relativistic). This system can be expanded so a similar beam pulse with twice the peak current is accelerated by $v_{0} / 2$ by two identical parallel strip lines, as shown in Fig. 3. The two transmission-line accelerator schemes in Figs. 2 and 3 do not require a switch to control the interaction of the transmission line and beam pulse. However, the change in beam potential is only one-half of the charging potential for these matched systems.
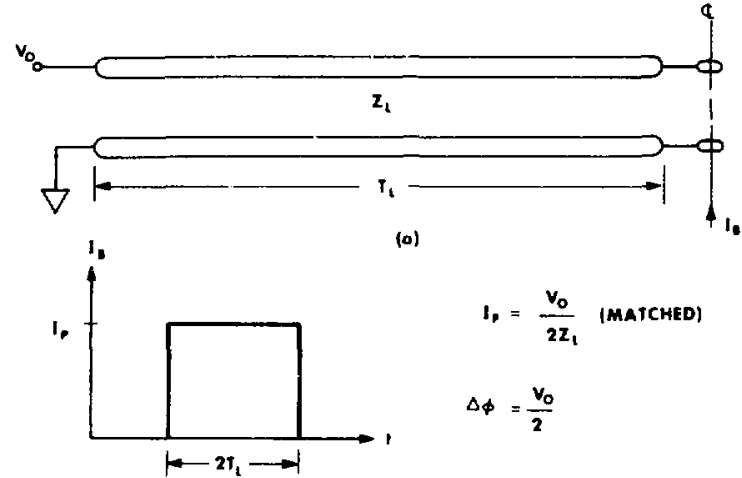

$$
\begin{aligned}
l_{1} & =\frac{V_{0}}{2 Z_{1}} \text { (MATCHED) } \\
\Delta_{\phi} & =\frac{V_{0}}{2}
\end{aligned}
$$

(b)

Fig. 2. Charged transmission-line accelerator.

The single Blumlein trairsmission-line generator on the left side of Fig. 4 is a standard inethod of achieving a voltage or potential output equal to the charging voltage. The Blumlein line does have a higher source impedance; therefore, the peak current is reduced to $I_{p}=V_{0} / 4 Z_{L}$ for matched operation. Also, a switch $S$ is required to control the interac$t$ ion of the accelerator with the beam pulse, as in the ETA/ATA system of Fig. 1. When switch $S$ is closed, a shoriting wave travels to the other end of the transmission line in a time $T_{L}$. Then a matched beam pulse is accelerated by a potential $V_{0}$ for a time $T_{P}=2 T_{L}$ as shown in Fig. 4. This circuit also can be paralleled as indicated by the dashed lines of Fig. 4. The peak current $I_{p}$, accelerated by potential $v_{0}$, can be doubled or $I_{p}=V_{0} / 2 Z_{L}$. The two Blumlein lines can be activated by a single switch $\sigma$ by separate switches closed simultaneously.
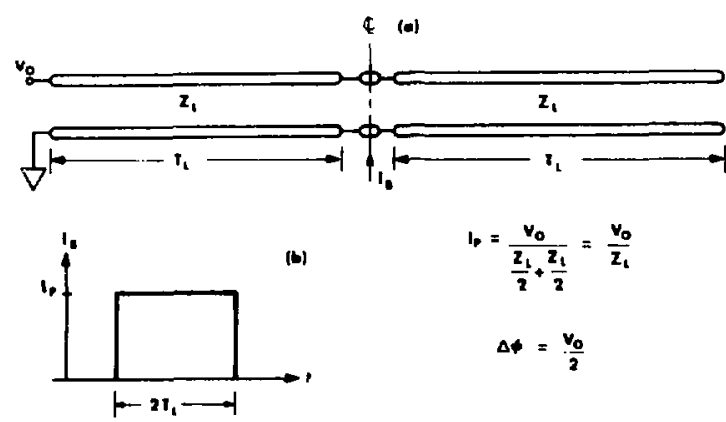

$$
\begin{gathered}
I_{p}=\frac{v_{0}}{\frac{z_{b}}{2}+\frac{z_{1}}{2}}=\frac{v_{0}}{Z_{1}} \\
\Delta t=\frac{v_{0}}{2}
\end{gathered}
$$

Fig. 3. Parallel charged transmission-line accelerator. 


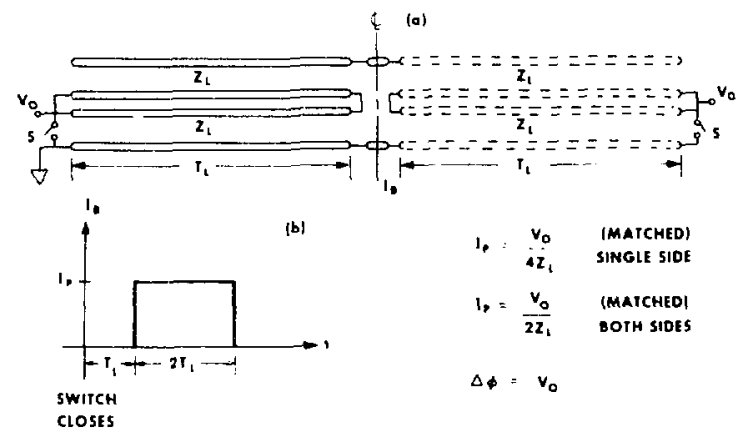

Fig. 4. blumiein transmission-line accelerators.

Uperation of a Blumlein line can be characterized as voltage-vector inversion. The sum of the voltage vectors from $A$ to $C$ in the generalized Blumlein line of Fig. ba is zero before the switch is closed at time $t=0$. If the Blumlein line is not loaded across $A$ and $C$ and the switch is closed at $t=0$, a voltage shorting wave propagates down the transmission line to the open circuit at terminals $A B$ and inverts the voltage vector in the bottom transmission line of Fig. 5a. When the transient wave returns to the switch at $t_{0}=2 T_{L}$, the voltage vector in the bottom line is inverted everywhere as shown in Fig. 5b. At time $t=2 T_{L}$, the voltage potential available across $A$ and $C$ for the entire line is $2 V_{0}$. If an accelerator structure were connected to $A$ and $C$, the inversion switch were opened, and $a$ matched beam pulse were injected $\left(I_{p}=V_{o} / 4 Z_{L}\right.$, $\left.T_{p}=2 T_{L} L\right)$ at $t=2 T_{L}$, the pulse would be accelerated through a potential of $V_{0}$ for a period of $2 T_{L}$ until $t=3 T_{L}$. Note that the perfect switch was opened in this example to make the accelerator operation compatible with transmission-line operation.

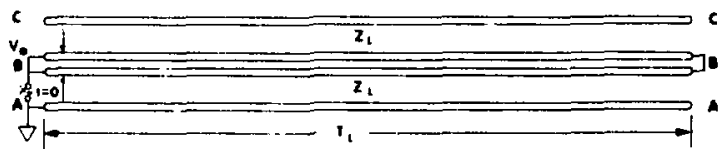

(a) VOLTAGE VECTONS AI IIME $=0$

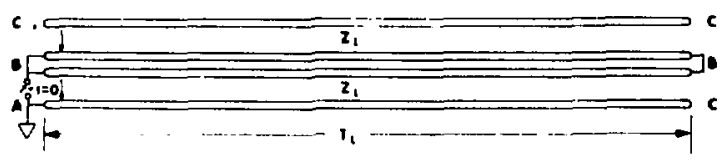

(b) VOUTAGE VECIORS AT TIME $=2 T_{1}$

Fig. b. Vector inversion of long transmission line.
This example is used only to illustrate the principle of voltage-vector inversion and its use in an accelerator system.

If the transmission lines of Fig. 5 are much longer than the pulse to be acceler ted and, in particular, an integral number of pulse iengths, $T_{L}=$ $m T_{p}$, several pulses can be accelerated in parallel by the same set of transmission lines as shown in Fig. $b$, where $m=3$. Note that this arrangement consists of three dual Blumlein line accelerators (previously illustrated in Fig. 4) connected in series, but with only one switch used for voltage inversion.

Operation of the system in Fig. 6 can be made consistent with transmission-line operation (no switch opening required) in the following manner. The two transmission lines are charged initially to voltage $v_{0}$. The switch is closed at $t=0$, and the voltage inversion takes place as noted for the system in Fig. 3. The accelerator structures in Fig. 6 must be designed to cause minimal discontinuities and, therefore, reflections of the inversion transients. Also, the accelerator structures must not be loaded (no beam pulse present) during the inversion process. After the inversion wave has reached the open end and returned to $A\left(t=5.5 T_{p}\right)$, matched beam pulses $\left(I_{p}=V_{o} / 4 Z_{L}, T_{p}=T_{L} / 3\right)$ are simultaneously injected at $A, B$, and $C$. These pulses are accelerated by a potential $V_{0}$ for a period of $T_{p}=T_{L} / 3$. The individual accelerator structures at $A, B$, and $C$ are isolated from each other by the transit time of the transmission line.

This particular arrangement offers several advantages. First, only one switch is used to accelerate three beam pulses. Second, the switch used only to invert the long transmission-line potential vectors does not have to be as high quality as a switch that is involved directly in coupling the accelerator to the beam. A third advantage, which is not immediately obvious, is the repetition-rate advantages of this puise system. The bottom transmission-line section in Fig. 6 from input $A$ to the switch can be lengthened and a transmission-line fanout can be added to multiplex several switches, one in each leg of the fan-out. This arrangement permits higher rate operation through easy switch multiplexing. The short-duration transients caused by 


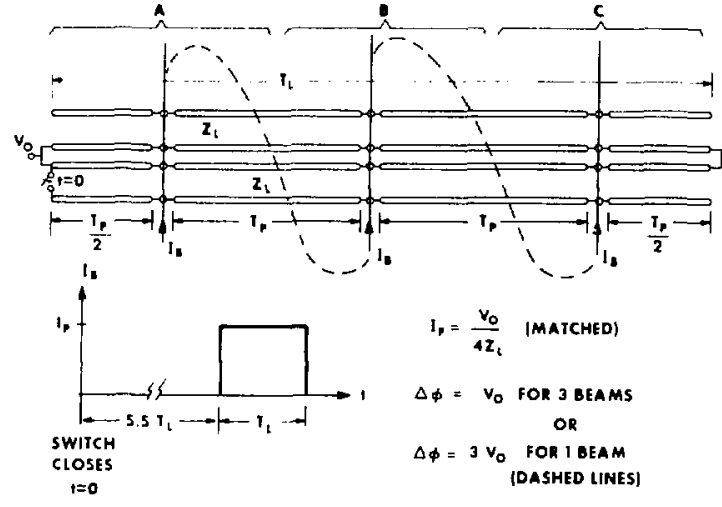

Fig. o. Transit-time ITLA.

the impedance discontinuities of the transmissionline fan-out will not affect the accelerator struc$t$ ures and process because of the time delay between switch closure and acceleration.

Another mode of operation is indicated in Fig. 6 by the dashed lines. If the transit time of an accelerated particle from the output of accelerator section $A$ to the input of section $B, T_{A B}$, could be made nuch less than the transmission-line transit time between structures of $T_{A B} \ll T_{p}$, a single beam could, in principle, be accelerated $m$ times $(m=3)$ by the same structure, and the beam trajectory generally would be helical through the structure.

Because of the practical difficulties of providing the relical beam trajectory and the impossibility of reducing $T_{A B} \ll T_{p}$ for this system, an alternative is to deform the transmission lines into a helix and provide a straight beam trajectory. This method also makes the condition $T_{A B} \ll T_{p}$ feasible, which is the essence of the helical edge-wound stripline accelerator (HEWSLA) concept. ${ }^{2}$

A major practical consideration in accelerator - ems involves enclosed potentials or grounded beam input and output ports. Another modification of the accelerator system in Fig. 6, shown in Fig. 7 , provides grounded beam input and output ports. Basically, inductive isolation separates the bottom line from the top line, and the opposite polarity acceleration potential is applied across this inductive isolation. The beam trajectory passes through a closed metal cylinder or $\boldsymbol{d}$ ift tube and does not experience the external decelerating field. The isolation inductance $L_{I}$ of this path is made large so

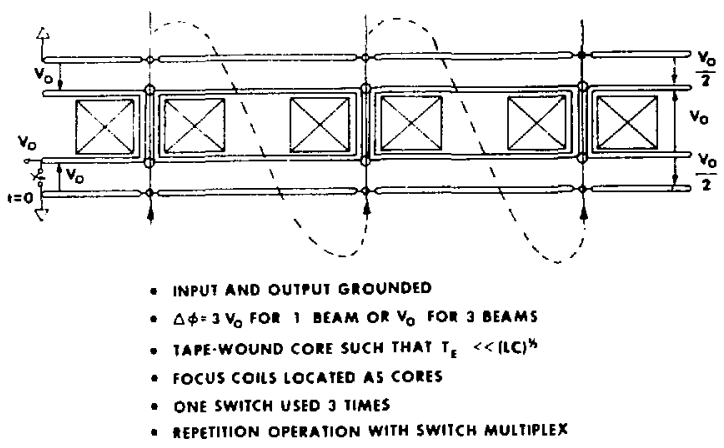

Fig. 7. Transit-time and inductance ITLA.

that the discharge time of the transmission-line capacitance $C_{L}$ is longer than the inversion time $T_{i}$, or $\pi\left(L_{I} C_{L}\right)^{1 / 2} \gg T_{i}=m T_{P}$.

The inductance is enlarged by using tape-wound ferromagnetic cores. In the electrical circuit of this scheme, the isolating inductance appears in parallel with the beam pulse load during acceleration. Thus, this scheme can be labeled an induction accelerator. However, this concept can use lower frequency response and higher saturation flux materials (steel instead of ferrite) than the ETA/ATAtype system can.

The inductive isolation can be combined with transit time isolation to accelerate m pulses simultaneously (parallel) or one pulse m times (series). A series ITLA module with $m=10$ is shown in Fig. 8 , and a parallel ITLA in which three pulses are accelerated simultaneously is shown in Fig. 9. The paraliel module could be used in an accelerator system that would series the ream pulses after acceleration with beam-handling techniques. In this context, the parallel ITLA can be considered a beam chopper.

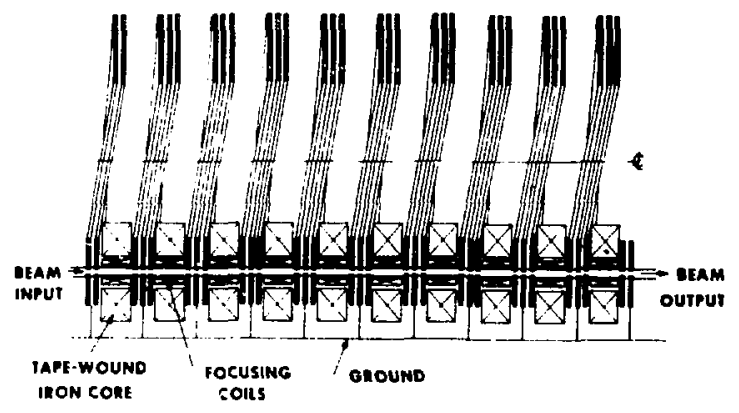

Fig. ช. Helical ITLA. 


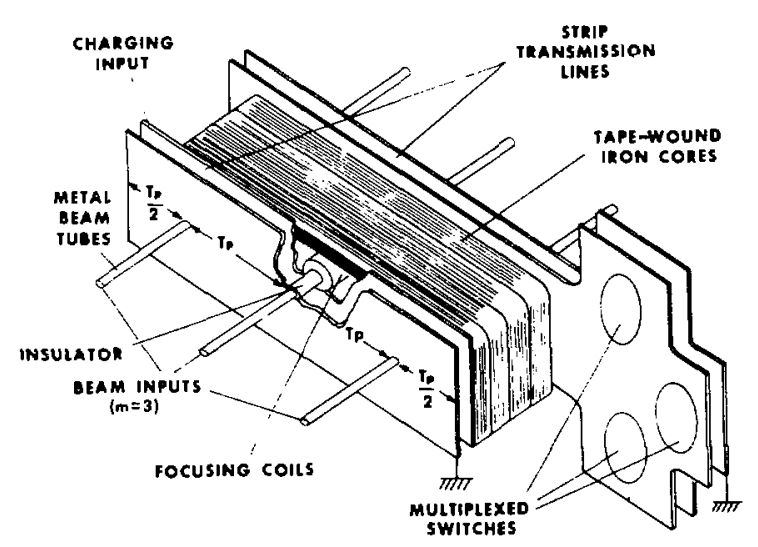

Fig. 9. Parallel ITLA.

\section{POINT OESIGNS}

To evaluate these concepts, a study of strip transmission-line parameters and other accelerator electrical concerns was undertaken for a series system similar to ETA/ATA. Basic strip transmissionline parameters are illustrated in Fig. 10. For a peak beam current $I_{p}$ and a stage acceleration voltage $V_{0}$, the source impedance $z_{s}$ must be

$$
Z_{s}=V_{0} / 2 I_{p}
$$

Because the ITLA system drives the beam from two sides or with two strip lines in parallel, the transmission-line impedance must be

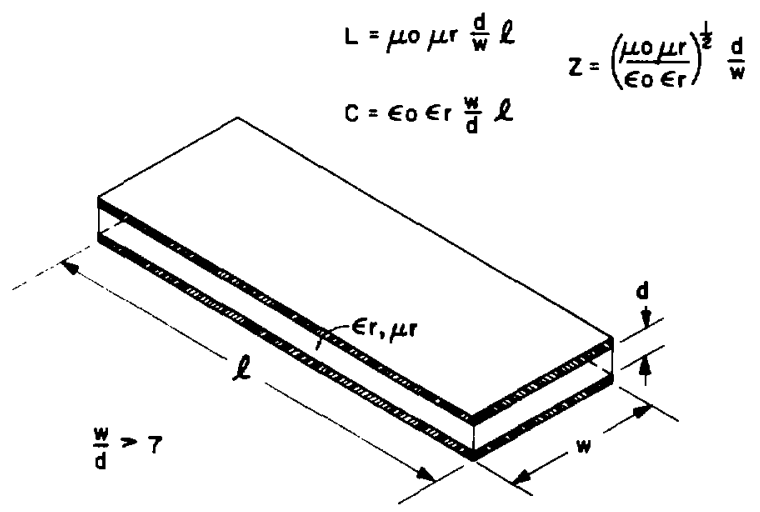

Fig. 10. Strip transmission-line fundamentals.

$$
L_{L}=2 Z_{s}=\frac{V_{o}}{I_{p}}=\frac{d}{W}\left(\frac{\mu_{0} \mu_{r}}{\varepsilon_{0} \varepsilon_{r}}\right)^{1 / 2},
$$

where $d$ is the line separation, $W$ is the transmission-line width, $\mu_{r}$ is the effective permeability of the core, $\mu_{0}$ is the permeability of free space, $\varepsilon_{0}$ is the permittivity of free space, and $\varepsilon_{r}$ is the dielectric relative permittivity.

The line separation is determined by the dielectric strength $k_{s}$ of the medium between the conductors or

$$
d \geqslant \frac{V_{0}}{K_{s}}
$$

From Eqs. (2) and (3) the transmission line width is given by

$$
W \geq \frac{I_{p}}{K_{s}}\left(\frac{\mu_{0} \mu_{r}}{E_{0} E_{r}}\right)^{1 / 2} \text {. }
$$

To minimize the difference in the transmussionline transit time between the inside and the outside radii, the major helix radius $R_{h}$ is chosen such that

$$
R_{h} \geqslant 10 W
$$

In addition, the number of pulses $m$ to be accelerated and the helix radius $R_{h}$ are iterated to obtain an integer value for $m$ in the equation relating the turn transit time $T_{L}$ to the beam pulse width $T_{p}$, or

$$
T_{L}=m T_{p}=\frac{2 \pi R_{h} \varepsilon_{r}^{1 / 2}}{C} \text {, }
$$

where $C$ is the speed of light in vacuum.

Next, the erection or total line-inversion time $T_{j}$ is limited by the loss characteristics of the system. The first constraint on $T_{i}$ and thus on the number of nelix turns is the resistive decay time $T_{r c}$ or 
$T_{i} \leqslant 0 . i T_{r c}$

The line capacitance per unit length is

$$
C_{L}=\frac{\varepsilon_{0} \varepsilon_{r} W}{d} \text {, }
$$

and the line shunt resistance per unit length is

$$
\mathrm{R}_{\mathrm{L}}=\frac{\rho_{\mathrm{d}}}{W},
$$

such that

$$
T_{r C}=R_{L} C_{L}=\rho_{0} \varepsilon_{r} .
$$

The inductance of one turn is

$$
\mathrm{L}_{\mathrm{T}}=\mu_{\mathrm{o}}\left(\frac{\mu_{\mathrm{r}}{ }^{A_{\mathrm{C}}}}{\ell_{\mathrm{C}}}+\frac{A_{\mathrm{s}}}{\ell_{\mathrm{S}}}\right) \mathrm{n}^{2}
$$

where $A_{c}$ is the core area, $\ell_{c}$ is the core path length, and $\mu_{r}$ is the effective permeability of the core. The term $A_{s} / l_{s}$ represents the stray inductance and $n$ is the number of turns through the core. For the series system $n=1$ and $\mu_{r} A_{c} / P_{c} \gg A_{s} \mu_{s}$,

$$
L_{T} \simeq \frac{\mu_{0} \mu_{r c} A_{C}}{l_{c}}
$$

where $\mu_{0}$ is the permeability of free space and $\mu_{r c}$ is the relative permeability of the core.

The minimum core area must be such that

$$
2 A_{C} B_{S}=2 V_{0} T_{i},
$$

$$
A_{c} \geq \frac{V_{0} T_{i}}{B_{s}}
$$

To minimize inductive loading, the product of core area and relative permittivity, $\mu_{r}{ }^{A_{c}}$, is chosen to permit the number of helical turns desired. The turn inductance through the core is now

$$
\mathrm{L}_{\mathrm{T}}=\frac{\mu_{\mathrm{c}} \mu_{\mathrm{rc}}{ }_{\mathrm{o}} \mathrm{T}_{1}}{\ell_{\mathrm{c}} \mathrm{B}_{\mathrm{S}}} \times
$$

where $\alpha$ is the increase in the $\mu_{r c} A_{c}$ product required to obtain the desired number of helical turns n. The magnetic length of the core can be approximated by

$$
l_{\mathrm{C}}=\left(\frac{\mathrm{OD}+\mathrm{ID}}{2}\right) \pi
$$

where $O D$ is the core outside diameter and ID is the core inside diameter. The LC resonant time is $f i-$ nally

$T_{L C}=\pi\left(L_{T} C_{T}\right)^{1 / 2}=\left[\frac{4 \pi \mu_{0} \mu_{r C} v_{0} R_{h} \varepsilon_{0} \varepsilon_{r} W \alpha}{(00+10) B_{S} d}\right]_{(17)}^{1 / 2}$, where $C_{T}$ is the capacitance of one helical turn. The inversion time $T_{i}$ is related to the number of helical turns and to the structure parameters by

$$
T_{i}=2 n T_{L}=\frac{4 n \pi R_{h} E_{r}^{1 / 2}}{C} .
$$

The inversion time must be such that

$$
T_{i} \leqslant 0.1 T_{r c}
$$

where $B_{S}$ is the magnetic material saturation flux density or or 


$$
n \leq \frac{0.1 \subset \rho E_{o} \varepsilon_{r}^{1 / 2}}{4 \pi R_{h}} \text {, }
$$

to avoid resistive loading during erection. The reduction of inductive loading is determined by

$$
\mathrm{T}_{i} \leqslant 0.1 \mathrm{~T}_{\mathrm{LC}}
$$

From Eqs. (17), (18), and (21),

$$
T_{i} \leqslant(0.1 \pi)^{2}\left[\frac{4 \pi \mu_{0} \mu_{r c} \varepsilon_{0} \varepsilon_{r} V_{0} W R_{h}}{(G D+I D) d B_{s}}\right] ;
$$

thus,

$n \leqslant 2(0.1 \pi)^{2} c\left[\frac{\mu_{0} \mu_{r c} \varepsilon_{0} E_{r}{ }^{1 / 2} v_{0} W \alpha}{(00+10) d B_{s}}\right]$

or

$$
\alpha \geqslant \frac{n(O D+I D) d B_{S}}{2(0.1 \pi)^{2}\left(u_{0} \mu_{r C} E_{0} E_{r}^{1 / 2} v_{0} w\right.} \text {. }
$$

If a reasonable value of $n$, the number of helical turns and half the number of switches to be replaced is chosen, the value of $\propto$ and the erection time can be determined.

Thus, all the parameters for a series ITLA structure can be determined. The values for an oilinsulated, 1U-kA accelerator are listed in Table I.

\section{v. CIRCUIT SIMULATION}

The operation of a three-turn ITLA was simulated using the NET- 2 circuit analysis program. ${ }^{3}$ The circuit model is shown in Fig. 11, where the rectangular boxes represent transmission-line sections, $V 1$ is the $250-k V$ charging supply, and $S 1$ is the erection switch. Inductors L19, L20, and L21 represent the turn-isolation inductance associated

\begin{tabular}{|c|c|c|}
\hline Line-charge voltage & (V) & $=2.5 E+05$ \\
\hline Beam current & (A) & $=1.0 E+U 4$ \\
\hline beam puise width & (s) & $=0.0 E-08$ \\
\hline Line separation & $(\mathrm{m})$ & $=3.1 E-02$ \\
\hline Line width & $(m)$ & $=3.1 E-U 1$ \\
\hline Helix radius & $(m)$ & $=3.1 E+00$ \\
\hline Line impedance & $(\Omega)$ & $=\angle . J E-U 1$ \\
\hline Dielectric strength & $(\mathrm{V} / \mathrm{m})$ & $=0.0 E+46$ \\
\hline Relative dielectric constant & & $=2.3 \mathrm{E}+00$ \\
\hline Number of parallel pulses & & $=2$ \\
\hline Multiple of mir in: $m$ fiux & & $=1.2 E+u 0$ \\
\hline Erection time & (s) & $=1.0 E-06$ \\
\hline$R_{:- \text {-istive decay time }}$ & (s) & $=2.0 E-01$ \\
\hline Core area & $\left(m^{2}\right)$ & $=2.0 \mathrm{E} \cdot \mathrm{O} 1$ \\
\hline Core width along beam & $(m)$ & $=2.9 E-01$ \\
\hline RC limit of number of turns & & $=1.0 E+05$ \\
\hline LC limit of number of turns & & $=0.0 E+40$ \\
\hline Maximum number of turns & & $=5.0 E+00$ \\
\hline Acceleration per module & (V) & $=2.5 E+$ \\
\hline
\end{tabular}
with the steel cores. Inductors L7, L9, L11, L24,
TABLE I

PARAMETERS FOR OIL-INSULATED SERIES ITLA MODULE

$\mathrm{L} 26$, and $\mathrm{L} 28$ represent the beam inductance and associated resistance. (NET $-c$ requires that each inductor have a specified resistance.) The computer-generated wave form in Fig. 12 illustrates the erection of the three-turn ITLA. The acceleration potential in Fig. $i 2$ is the sum of the voltages across the

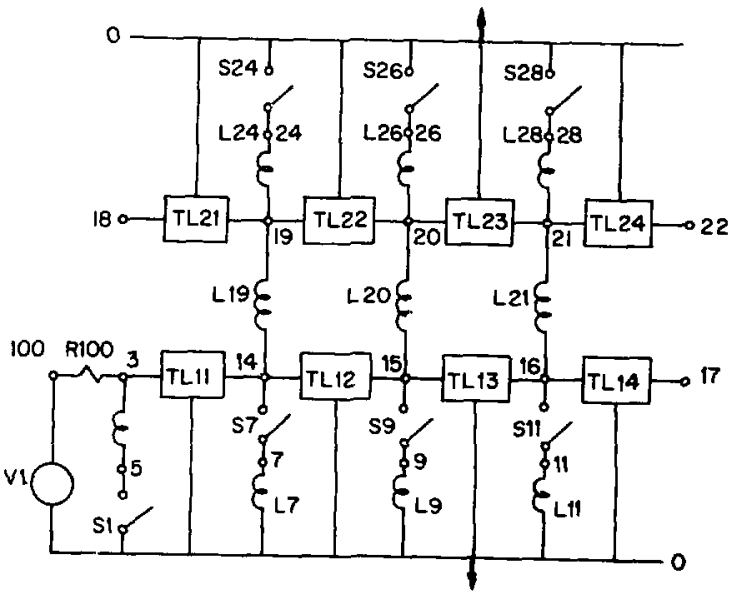

Fig. 1i. Three-turn series ITLA circuit model. 


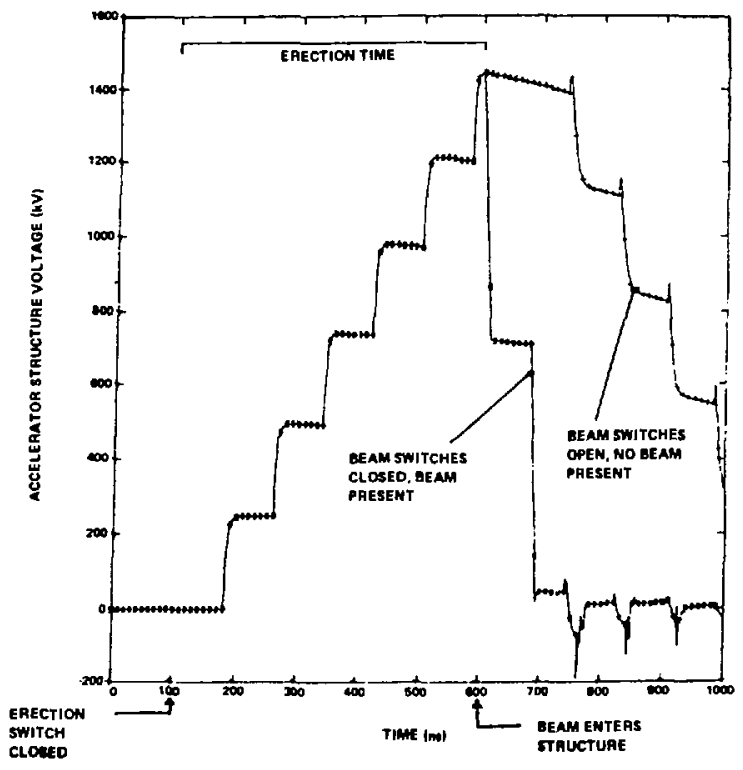

Fig. 12. Three-turn series ITLA erection and discharge wave forms.

transmission line at each acceleration port. Initially, the sum of the charge voltages equals zero. After the main switch (S1) is closed, the wave transients passing by the beam switches invert the bottom line so that the peak erection voltage is $1500 \mathrm{kV}$ or $\mathrm{cm}(\mathrm{m}=3)$ times the charge voltage of $250 \mathrm{kV}$. If the beam switches are closed at peak voltage ( $t=000 \mathrm{~ns})$, a majority of the transmissionline energy is delivered to the load resistors (associated with load inductances). The effective current through one beam load section is shown in Fig. 13, and the acceleration potential imparted to the beam is shown in Fig. 14. Note that the highfrequency spikes in the circuit sinulation are due to the ideal transmission-line sections. The circuit simulation can be considered a first-order approximation. For example, all the beam switches were closed simultaneously, but in reality they should be staggered to represent the beam pulse traveling through the accelerator structures in series.

\section{CONCLUSIONS}

Isolated transmission lines can be used to reduce the number, quality, and requirements of highpower switches in pulsed accelerators. The param-

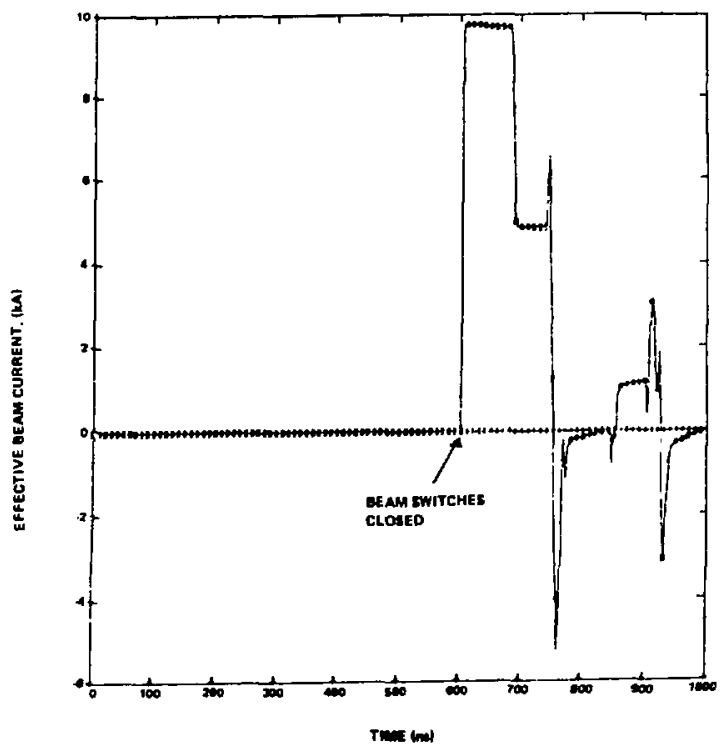

Fig. 13. Three-turn series ITLA effective beam current.

eters in Table I indicate that the number of switches in an ETA/ATA-type system can be reduced 10 times for a symmetrical series ITLA in which the erection switch is in the center of a 10-turn helix. Operation of the switches in repetition mode is

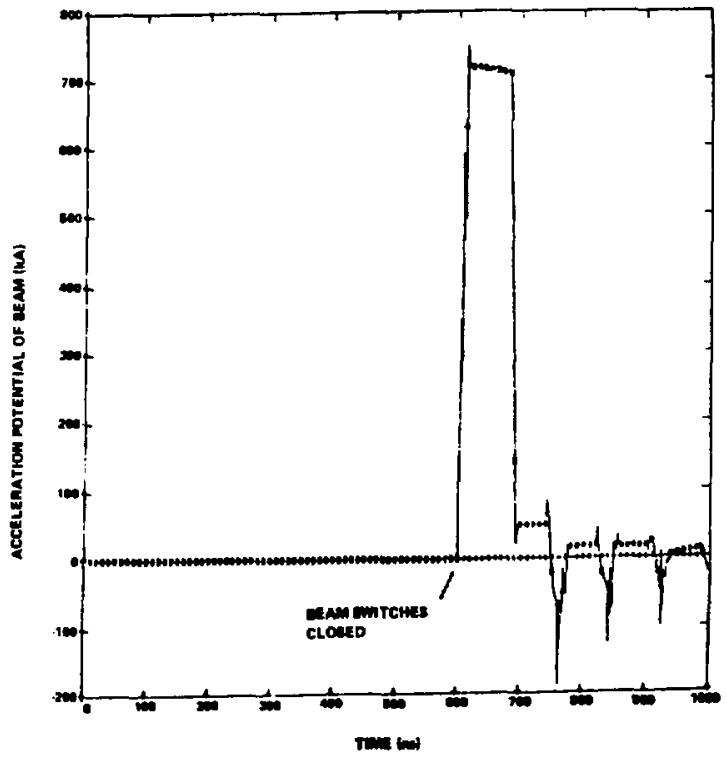

Fig. 14. Three-turn series ITLA acceleration potential. 
facilitated in the ITLA sysiem because timing and switching requirements are reduced. Switch multiplexing also is facilitated by the use of switches in a secondary rather than a primary role.

\section{REFERENCES}

1. R. E. Nester, D. G. Bubp, J. C. Clark, A.W. Chesterman, E. G. Cook, W. L. Dexter, et al.,
"The Experimental Test Accelerator (ETA)," IEEE Trans. Nuc 1. Sci. NS-26, (3) 4180-4162 (June 1979).

2. W. C. Nunnally, "HEWSLA--He lical Edge-Wound Strip-Line Accelerator," Los Alamos National Laboratory internal report (March 1979).

3. Allan F. Ma lmberg, "NET-2 Network Analys is Program," Release 9.1 , Braddock, Dunn and McDonald, Inc. (September 1979). 